\title{
Droplet digital PCR for the detection and monitoring of Legionella pneumophila
}

\author{
LUCA FALZONE $^{1 *}$, GIUSEPPE GATTUSO $^{2 *}$, CINZIA LOMBARDO $^{3}$, GABRIELLA LUPO $^{4}$, \\ CATERINA MARIA GRILLO ${ }^{5}$, DEMETRIOS A. SPANDIDOS ${ }^{6}$, MASSIMO LIBRA $^{2,7}$ and MARIO SALMERI ${ }^{3,7}$ \\ ${ }^{1}$ Epidemiology Unit, IRCCS Istituto Nazionale Tumori ‘Fondazione G. Pascale’, I-80131 Naples; \\ ${ }^{2}$ Department of Biomedical and Biotechnological Sciences, Section of General Pathology, University of Catania; \\ ${ }^{3}$ Department of Biomedical and Biotechnological Sciences, Section of Microbiology, University of Catania; \\ ${ }^{4}$ Department of Biomedical and Biotechnological Sciences, Section of Biochemistry, University of Catania; ${ }^{5}$ Department of \\ Medical Sciences, Surgical and Advanced Technologies, GF Ingrassia, University of Catania, I-95123 Catania, Italy; \\ ${ }^{6}$ Laboratory of Clinical Virology, Medical School, University of Crete, 71003 Heraklion, Greece; \\ ${ }^{7}$ Research Center for Prevention, Diagnosis and Treatment of Cancer, University of Catania, I-95123 Catania, Italy
}

Received August 13, 2020; Accepted September 14, 2020

DOI: $10.3892 /$ ijmm.2020.4724

\begin{abstract}
Legionella pneumophila (L. pneumophila) is a harmful pathogen often found in water systems. In hospitals, the absence of L. pneumophila in water systems is mandatory by law, therefore, frequent and effective monitoring of water is of fundamental importance. Molecular methods based on reverse transcription-quantitative polymerase chain reaction (RT-qPCR) have been proposed for the detection of L. pneumophila, however, the sensitivity and accuracy of these methods have not been validated yet. Therefore, it is important to evaluate other strategies able to overcome the limits of culture-based and RT-qPCR methods. On these bases, we compared the sensitivity and accuracy of droplet digital PCR (ddPCR) and RT-qPCR in water samples with known concentrations of L. pneumophila and in an in vitro model of water heat treatments. ddPCR showed a higher sensitivity rate and accuracy compared to RT-qPCR in detecting low bacterial load. In addition, ddPCR is not affected by the presence of fragmented DNA and showed higher accuracy than RT-qPCR in monitoring the efficacy of heat shock treatments. In conclusion, ddPCR represents an innovative strategy to effectively detect L. pneumophila in water samples. Thanks to its high robustness, ddPCR could be applied also for the detection of L. pneumophila in patients with suspected legionellosis.
\end{abstract}

Correspondence to: Professor Massimo Libra, Department of Biomedical and Biotechnological Sciences, Section of General Pathology, University of Catania, I-95123 Catania, Italy

E-mail:m.libra@unict.it

${ }^{*}$ Contributed equally

Key words: Legionella pneumophila, ddPCR, RT-qPCR, diagnosis, sensitivity, molecular biology

\section{Introduction}

Legionella are aerobic, environmental, gram-negative bacteria accounting for 61 different strains and approximately 70 serogroups; some of these strains are able to induce severe pathological manifestations in humans. The name 'Legionella' derives from a group of veterans of the American Legion that in 1976 contracts the infection during a stay in a Hotel in Philadelphia where an outbreak of Legionella was present in the air conditioning system. This outbreak caused 34 deaths among 221 infected, however, only one year later the causative agent of infections was recognized and named as Legionella pneumophila (L. pneumophila) (1).

L. pneumophila accounts for 35 different serogroups of which L. pneumophila serogroup 1 is the most frequently identified in Legionella infections (2). There are two clinical manifestations of legionellosis: the first form is a self-limiting disease, called Pontiac fever, that does not imply lung involvement, while the second form is called Legionnaires' disease that causes a pneumonia characterized by fever (in some cases even higher than $40^{\circ} \mathrm{C}$ ), chills, coughs, chest pain and in some cases by extrapulmonary symptoms such as diarrhea, nausea, vomiting, and neurological manifestations (1).

Legionellosis is a global health problem; according to the data provided by the European Epidemiological Report of the European Center for Disease Prevention and Control (ECDC) in $2017,9,238$ cases were reported, approximately $30 \%$ more infections than recorded in 2016 (3). An increasing trend was also found in Italy, where in 2017, 2,014 cases were reported, corresponding to a rate of 33.2 cases per million inhabitants, compared to the previous year where the incidence was 28.2 cases per million inhabitants. The mortality rate calculated on all cases with known outcome is $10.1 \%$ (4).

As mentioned, Legionella is widely spread in nature, in both natural and artificial water habitats. In particular, water systems, cooling towers and hydric pipeline are the ideal environment for its proliferation representing potential reservoir 
of infection. In addition, Legionella prefers warm habitats with ideal temperature ranging from $25^{\circ} \mathrm{C}$ to $42^{\circ} \mathrm{C}$ (under $20^{\circ} \mathrm{C}$, Legionella survives in a latent state). On this basis, it is possible to identify areas that due to the recirculation of hot water, such as water heaters, whirlpools, boilers, etc. can represent critical points of contamination $(1,5,6)$. In these favorable environments the conditions of development are optimal for the constant proliferation of Legionella and are potentially favorable also for biofilm formation $(7,8)$.

L. pneumophila is not transmissible from person to person but occurs after inhalation of contaminated aerosol droplets (9).

Therefore, the monitoring and management of all infrastructures and water systems are extremely important. Indeed, some operating conditions or structural characteristics of pipelines and water heaters can favor the growth and spread of Legionella. Guidelines have been issued in order to propose standard methods for the monitoring of legionellosis. Such guidelines contain the methods and indications necessary to guarantee levels of acceptability of Legionella in the putative sites at risk of contamination. In particular, various methods of prevention and control of contamination are indicated, such as water chlorination and heat treatment. A key point of existing guidelines is the time point of checks that have to be performed periodically in order to assess the effectiveness of the treatments or to establish the need to carry out further interventions $(10,11)$.

As reported in the ISO 11731:2017 protocol, the main method for detecting Legionella in environmental samples is represented by the culture-based method that allows to detect the presence and quantity of bacteria present in the analyzed samples (11). However, this method presents some problems, represented by rather long waiting times due to the low growth rate of L. pneumophila. Furthermore, in some cases, the culture method is impractical as the growth of Legionella can be inhibited by the presence of other bacteria (12), making it necessary to pre-treat the sample in order to avoid growth inhibition or bacterial contamination $(13,14)$.

More recently, molecular identification methods have been proposed to overcome the main problems of culture-based methods. Among these molecular techniques, reverse transcription-quantitative polymerase chain reaction (RT-qPCR) allows the identification of Legionella in environmental samples faster than culture methods (15-17). In addition, this method has a greater sensitivity than the cultural examination, however, RT-qPCR is not able to distinguish between viable and non-viable organisms (18).

It is evident that the possibility of having a rapid evaluation of the number of live or dead microorganisms present in water samples is of fundamental importance for the maintenance of public health. The use of highly sensitive methods especially in high-risk environment, such as hospitals, hospice or nursing homes for the elderly, are fundamental in order to avoid outbreak of legionellosis. Indeed, in hospitals the limits established by law indicate that the microbial load of Legionella is below 102 CFU/1, especially in those Departments with immunosuppressed patients (e.g., Intesive Care Units, Medical Oncology Units, etc.) where the total absence of L. pneumophila is mandatory (10). Therefore, in these high-risk environments it is of fundamental importance to implement effective strategies to monitor and control the onset of possible contamination. In this context, culture-based methods fail to promptly identify possible outbreaks of infection due to the low growth rate of L. pneumophila. Therefore, there is an urgent need to standardize novel methods for the early and rapid identification of contaminated sites.

On these bases, the aim of the present study is to propose droplet digital PCR (ddPCR) as a novel high-sensitive method for the rapid detection of L. pneumophila. For this purpose, ddPCR and RT-qPCR were used for the detection of L. pneumophila in an in vitro model of water tank contaminated with a known concentration of L. pneumophila. The sensitivity and accuracy of ddPCR and RT-qPCR were also tested by simulating water heat shock treatments in order to assess and validate the clinical application of ddPCR for the early diagnosis of Legionellosis.

\section{Materials and methods}

Bacterial strain and culture conditions. L. pneumophila serotype 1 was obtained from an environmental contaminated water site. Serotype 1 was identified by using lactic test (Oxoid, Cambridge, UK). L. pneumophila was grown on liquid broth and then on GVPC medium (Oxoid), corresponding to buffered charcoal yeast extract medium plus antimicrobial agents, at $37^{\circ} \mathrm{C}$ in a $5 \% \mathrm{CO}_{2}$-enriched atmosphere for 5 days. After growth, single colonies were resuspended in sterile water until a turbidity of $0.5 \mathrm{McF}$ arland $\left(1.5 \times 10^{8} \mathrm{CFU} / \mathrm{ml}\right)$ was obtained.

L. pneumophila dilution and DNA extraction. A dilution of $10^{7} \mathrm{CFU} / \mathrm{ml}$ was obtained starting from a concentration of $1.5 \times 10^{8} \mathrm{CFU} / \mathrm{ml}$. Then, 10 -fold serial dilutions in sterile water were performed until a concentration of $10 \mathrm{CFU} / \mathrm{ml}$ was obtained. The 10 -fold serial dilutions were used to assess the sensitivity of both methods.

In parallel, L. pneumophila was seeded into two flasks with sterile water with a final concentration $10^{2} \mathrm{CFU} / \mathrm{ml}$. One of the two flasks was subjected to thermal shock at $80^{\circ} \mathrm{C}$ for $30 \mathrm{~min}$ for 3 consecutive days in order to kill L. pneumophila. Different samples were obtained at different time points from the two flasks in order to assess the efficacy of thermal shock and the sensitivity of both ddPCR and RT-qPCR (Fig. 1).

Then, $1 \mathrm{ml}$ of each dilution or sample obtained from the two flasks was extracted using the PureLink Genomic DNA Mini kit extraction kit following the manufacturer's instructions (cat. no. K1820-01; Invitrogen; Thermo Fisher Scientific, Inc.). The extracted DNA was quantified by using spectrophotometric assay (Nanodrop 1000; Thermo Fisher Scientific, Inc.).

L.pneumophila RT-qPCR and ddPCR amplification. Extracted DNA $(4.7 \mu \mathrm{l})$ was amplified by using both SYBR-Green RT-qPCR and EvaGreen ddPCR.

For RT-qPCR, the Luminaris Color HiGreen qPCR Master Mix, high ROX (Thermo Fisher Scientific, Inc.) was used according to the manufacturer's protocol. L. pneumophila were amplified with a 7300 Real-Time PCR System (Applied Biosystems; Thermo Fisher Scientific, Inc.) using the following primer pairs and thermal conditions: forward: AGGGTTGATAGGTTAAGAGC; reverse: 


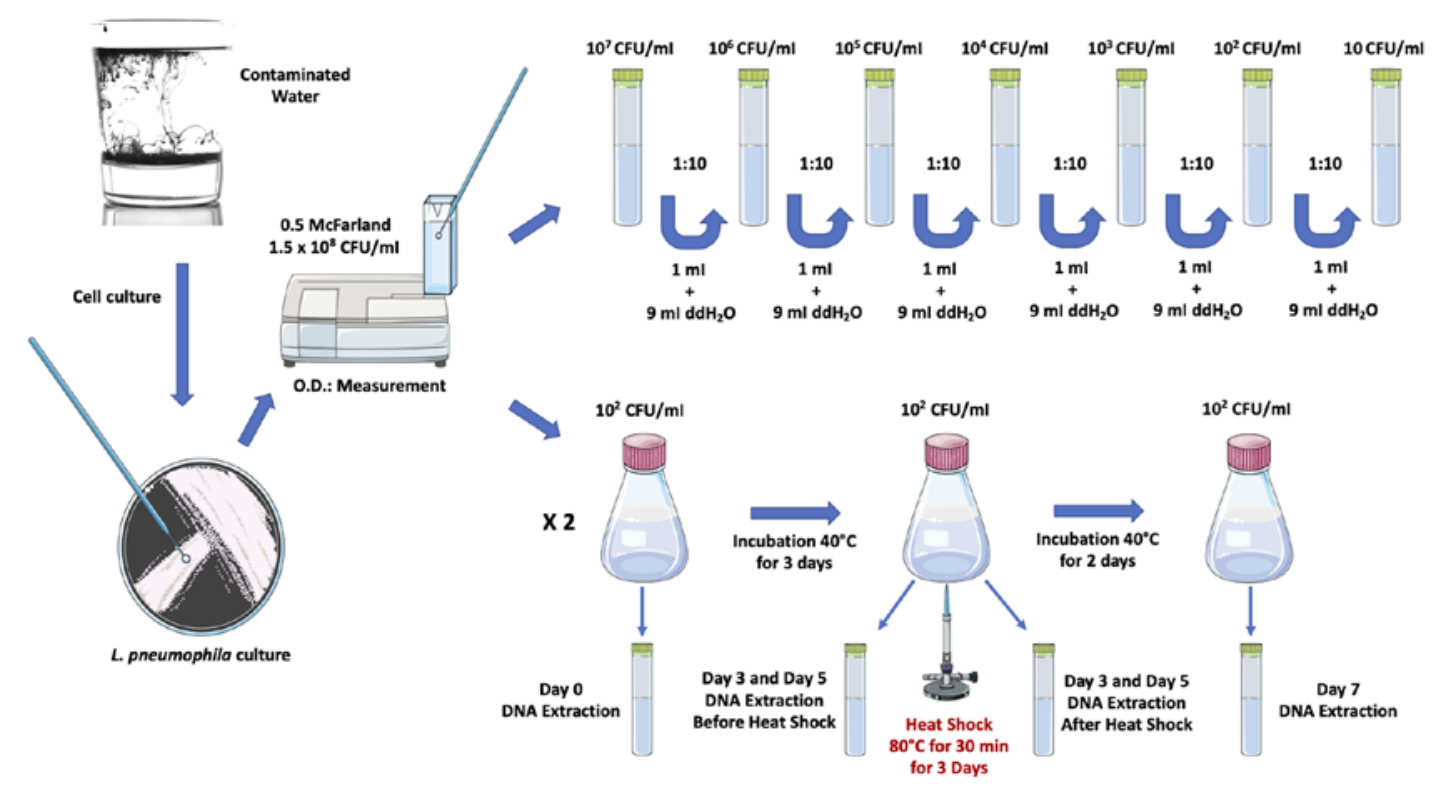

Figure 1. Schematic representation of the experimental design.

CCAACAGCTAGTTGACATCG; RT-qPCR thermal profile: UDG pre-treatment at $50^{\circ} \mathrm{C}$ for $2 \mathrm{~min}$, followed by an initial denaturation step at $95^{\circ} \mathrm{C}$ for $10 \mathrm{~min}$ and a 3-step PCR program at $95^{\circ} \mathrm{C}$ for $15 \mathrm{sec}, 60^{\circ} \mathrm{C}$ for $30 \mathrm{sec}$ and $72^{\circ} \mathrm{C}$ for $30 \mathrm{sec}$, for 40 cycles. The selected primers are specific for all L. pneumophila serogroups.

For EvaGreen ddPCR, the reaction mix was prepared by using $11 \mu 1$ of $2 \mathrm{X}$ QX200 ${ }^{\mathrm{TM}}$ ddPCR $^{\mathrm{TM}}$ EvaGreen Supermix (cat. no. 1864034; Bio-Rad Laboratories, Inc.), $0.15 \mu 1$ of $20 \mu \mathrm{M}$ forward and reverse primers (same primers used for RT-qPCR), $6 \mu 1$ of RNase and DNase free-water and $4.7 \mu 1$ of cDNA in order to obtain a final volume of $22 \mu l$.

Twenty microliters of the reaction mix were used to generate droplets with the QX200 droplet generator (BioRad Laboratories, Inc.). After generation, the droplets were transferred into a 96-well plate, sealed and amplified in a C1000 Thermal Cycler (Bio-Rad Laboratories, Inc.) under the following thermal conditions: polymerase activation at $95^{\circ} \mathrm{C}$ for $10 \mathrm{~min}, 40$ cycles of amplification at $94^{\circ} \mathrm{C}$ for $30 \mathrm{sec}$ (denaturation) and $60^{\circ} \mathrm{C}$ for $1 \mathrm{~min}$ (annealing/elongation), droplets stabilization at $98^{\circ} \mathrm{C}$ for $10 \mathrm{~min}$ followed by an infinite hold at $4^{\circ} \mathrm{C}$. A ramp rate of $2^{\circ} \mathrm{C} / \mathrm{sec}$ was used among the steps of the amplification.

Statistical analysis. The ddPCR data were statistically analyzed by using the QuantaSoft software provided by Bio-Rad Laboratories, Inc. Linear regression analysis was performed by using GraphPad Prism V.6 (GraphPad Software, Inc.). $\mathrm{P}<0.05$ was considered to indicate a statistically significant difference. All experiments were performed in triplicate.

\section{Results}

Comparison of specificity of $d d P C R$ and RT-qPCR. The serial dilutions obtained were used to assess the sensitivity of both ddPCR and RT-qPCR systems by using EvaGreen and SYBR-Green technologies, respectively. The obtained results showed that both methods are sensitive enough to detect the presence of L. pneumophila at concentrations established by law $\left(10 \mathrm{CFU} / \mathrm{ml}\right.$, i.e., $10^{2} \mathrm{CFU} / \mathrm{l}$ if the standard protocol for L. pneumophila detection starting from a 1 liter filtered water sample is used) (10). However, the signal related to the sample diluted at $10 \mathrm{CFU} / \mathrm{ml}$ was obtained at a very late $\mathrm{Ct}$ value (31.10) when RT-qPCR is used, while ddPCR effectively detects as positive the signals obtained for the same concentration (Table I; Fig. S1).

Noteworthy, the absolute quantification performed by ddPCR showed that the copies $/ \mu 1$ obtained for each sample better reflects the 10 -fold serial dilutions performed, except for the concentration of $10^{7} \mathrm{CFU} / \mathrm{ml}$ that was underestimated due to the high number of positive droplets that saturated the ddPCR system (Fig. S1A and B). In addition, linear regression analysis showed that ddPCR has a greater accuracy and robustness compared to RT-qPCR. By excluding the $10^{7} \mathrm{CFU} / \mathrm{ml}$ concentration that saturated both ddPCR and RT-qPCR systems, linear regression analysis revealed that ddPCR has a better $\mathrm{r}^{2}$ coefficient compared to RT-qPCR $\left(r^{2}=0.8388\right.$ vs. $\left.r^{2}=0.5228\right)$ (Fig. 2$)$.

ddPCR shows higher accuracy than RT-qPCR in monitoring the efficacy of thermal shock. Although ddPCR and RT-qPCR showed similar sensitivity in the detection of L. pneumophila, the simulation of heat shock treatment in an in vitro contaminated water tank highlighted the important differences existing between methods. Indeed, RT-qPCR detected false-positive signals in the sample treated at $80^{\circ} \mathrm{C}$ for three days probably due to cell debris and residual degraded DNA that produced a nonspecific amplification signal. In addition, no significant variation was observed between the untreated samples after one day and three days of growth (Ct values of 22.00 and 23.06, respectively) (Table II; Fig. S2).

On the contrary, ddPCR effectively identify as positive all the untreated samples and as negative the samples shocked at $80^{\circ} \mathrm{C}$ both after one day and three days of treatment. Moreover, ddPCR finely detected slight variation in the number of $L$. pneumophila after one day, three days and 
Table I. RT-qPCR Ct values and ddPCR L. pneumophila absolute quantification.

\begin{tabular}{lccrr}
\hline & \multicolumn{2}{c}{ RT-qPCR } & \multicolumn{2}{c}{ ddPCR } \\
\cline { 2 - 3 } No. & Sample & $\mathrm{Ct}$ & Sample & Copies $/ \mu 1$ \\
\hline 1 & $10^{7} \mathrm{CFU} / \mathrm{ml}$ & 11.26 & $10^{7} \mathrm{CFU} / \mathrm{ml}$ & 2,298 \\
2 & $10^{6} \mathrm{CFU} / \mathrm{ml}$ & 15.02 & $10^{6} \mathrm{CFU} / \mathrm{ml}$ & 1,762 \\
3 & $10^{5} \mathrm{CFU} / \mathrm{ml}$ & 17.75 & $10^{5} \mathrm{CFU} / \mathrm{ml}$ & 913 \\
4 & $10^{4} \mathrm{CFU} / \mathrm{ml}$ & 22.62 & $10^{4} \mathrm{CFU} / \mathrm{ml}$ & 9.30 \\
5 & $10^{3} \mathrm{CFU} / \mathrm{ml}$ & 25.64 & $10^{3} \mathrm{CFU} / \mathrm{ml}$ & 1.40 \\
6 & $10^{2} \mathrm{CFU} / \mathrm{ml}$ & 28.06 & $10^{2} \mathrm{CFU} / \mathrm{ml}$ & 0.29 \\
7 & $10 \mathrm{CFU} / \mathrm{ml}$ & 31.10 & $10 \mathrm{CFU} / \mathrm{ml}$ & - \\
8 & $\mathrm{NTC}$ & - & $\mathrm{NTC}$ & \\
\hline
\end{tabular}

RT-qPCR, reverse transcription-quantitative polymerase chain reaction; ddPCR, droplet digital PCR; L. pneumophila, Legionella pneumophila

Table II. RT-qPCR Ct values before and after heat shock treatment.

\begin{tabular}{llc}
\hline No. & \multicolumn{1}{c}{ Sample } & RT-qPCR Ct \\
\hline 1 & $10^{2} \mathrm{CFU} / \mathrm{ml}$ day 1 no shock & 29.72 \\
2 & $10^{2} \mathrm{CFU} / \mathrm{ml}$ day 3 no shock & 28.51 \\
3 & $10^{2} \mathrm{CFU} / \mathrm{ml}$ day 3 post shock & - \\
4 & $10^{2} \mathrm{CFU} / \mathrm{ml}$ day 5 no shock & 25.35 \\
5 & $10^{2} \mathrm{CFU} / \mathrm{ml}$ day 5 post shock & $35.18^{\mathrm{a}}$ \\
6 & $10^{2} \mathrm{CFU} / \mathrm{ml}$ day 7 post shock & - \\
7 & NTC & - \\
\hline
\end{tabular}

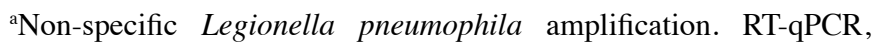
reverse transcription-quantitative polymerase chain reaction.

five days of growth without thermal treatments. In particular, after three days of incubation the number of copies increased from 1.9 to 5.3 copies $/ \mu 1$, while after 5 days of incubation the concentration increased at $25.5 \mathrm{copies} / \mu 1$ thus passing from an initial concentration of $10^{2} \mathrm{CFU} / \mathrm{ml}$ to a concentration of $2.7 \times 10^{3} \mathrm{CFU} / \mathrm{ml}$ (Fig. 3). Of note, no nonspecific signals were observed for the sample treated at $80^{\circ} \mathrm{C}$ for three days, suggesting that ddPCR is less prone to interference from degraded DNA or cellular debris.

\section{Discussion}

Different studies have demonstrated the higher sensitivity of ddPCR compared to RT-qPCR $(19,20)$. At present, ddPCR is one of the most sensitive methods used for the detection of low amounts of targets, including circulating DNA, microRNAs, circulating mutations, rare copy number variants, low viral nucleic acid targets representing a promising technology for use in clinical practice and in public and environmental health (21-24). Several studies have tried to propose and validate RT-qPCR-based molecular methods for the detection of L. pneumophila in contaminated water samples or for the diagnosis of Legionellosis in patients with suspected pneumonia, however, the sensitivity of the technique and the

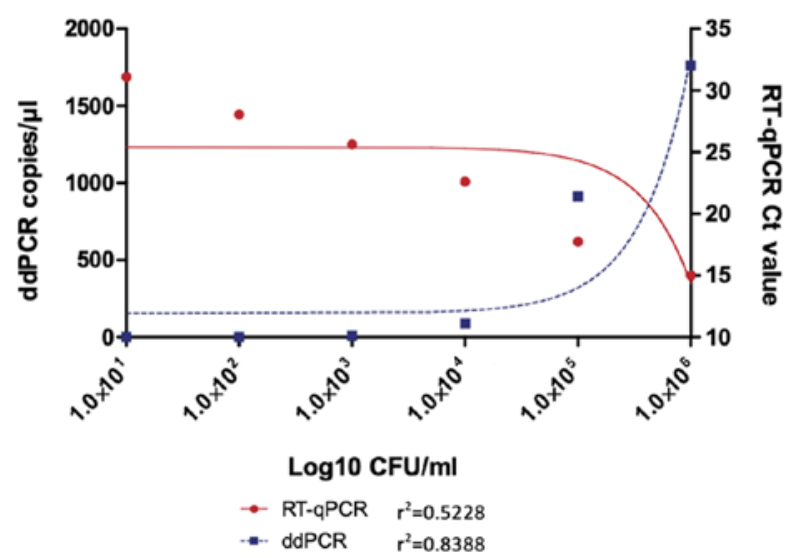

Figure 2. ddPCR and RT-qPCR linear regression analysis of L. pneumophila dilutions. ddPCR, droplet digital PCR; RT-qPCR, reverse transcription-quantitative polymerase chain reaction; L. pneumophila, Legionella pneumophila.

presence of inhibitor or contaminants may produce false-positive and false-negative results $(18,25,26)$. In order to overcome the limitations of RT-qPCR and to propose novel effective methods for L. pneumophila identification, here we compared the sensitivity and accuracy of ddPCR compared to RT-qPCR in detecting low levels of L. pneumophila and in monitoring the efficacy of water treatments.

The results here obtained demonstrated that both ddPCR and RT-qPCR have a good sensitivity, however, high-sensitive RT-qPCR detected low concentration of L. pneumophila at a very late $\mathrm{Ct}$ value (Ct 31.10 for $10 \mathrm{CFU} / \mathrm{ml}$ concentration). On the contrary, ddPCR accurately identified low concentrations of L pneumophila allowing absolute quantification of the bacterial load.

Moreover, interesting data were obtained by using both RT-qPCR and ddPCR for the evaluation of the efficacy of heat shock treatment. Indeed, RT-qPCR detected a false-positive sample after three days of heat treatments probably due to the presence of L. pneumophila cell debris and fragmented DNA that produced a nonspecific signal while ddPCR recognized as negative all the heat shock treated samples. Furthermore, the results obtained showed that ddPCR may be 


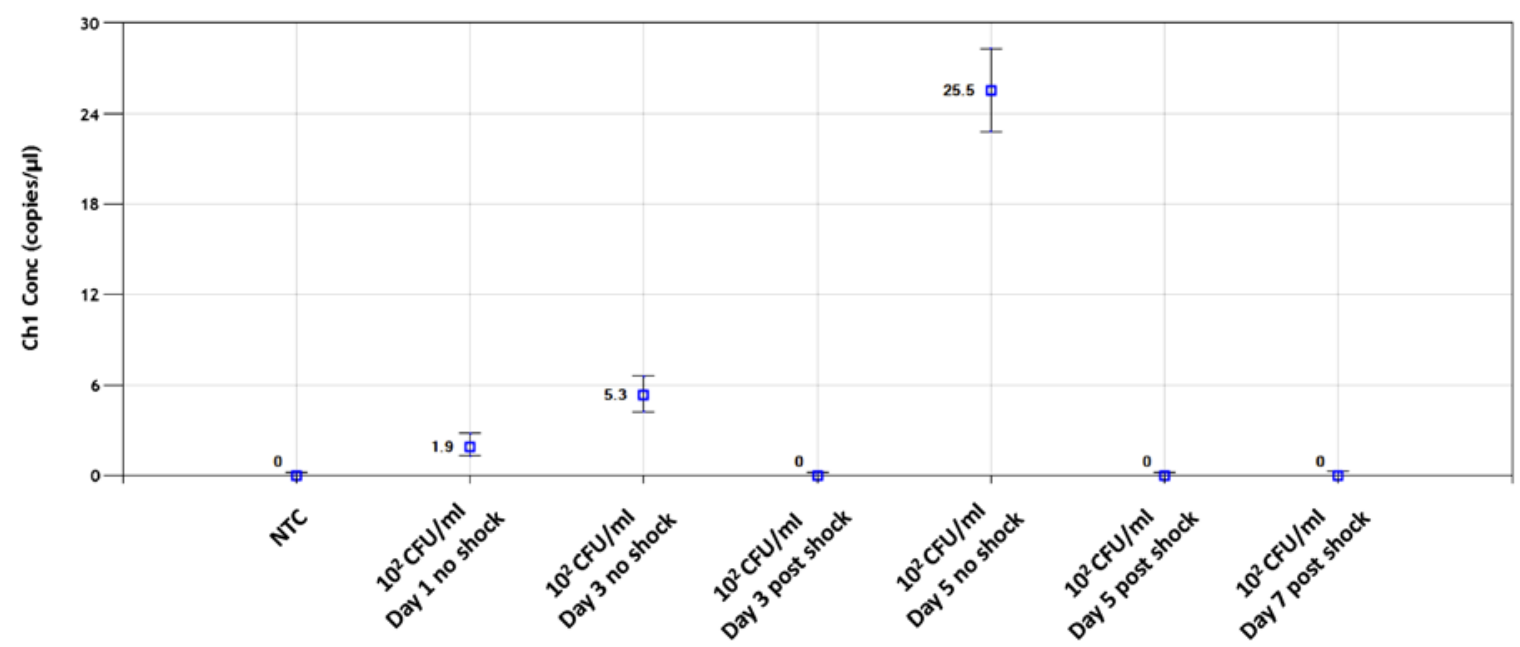

Figure 3. EvaGreen ddPCR absolute quantification (copies/ $/$ l) of $L$. pneumophila in heat shock untreated and treated samples. ddPCR, droplet digital PCR; L.pneumophila, Legionella pneumophila.

used also to detect weak increment of bacterial load in very limited time frames. Actually, ddPCR precisely detected the increase of L. pneumophila concentration after 3 and 5 days of growth without heat shock treatments. In particular, the initial concentration of 1.9 copies/ $\mu 1$ reached a concentration of $5.3 \mathrm{copies} / \mu 1$ (more than 2.5 -fold higher) after three days of growth, an increase that had not been identified by RT-qPCR. After 5 days of incubation L. pneumophila reached a concentration of 25.5 copies $/ \mu$ l. These results suggest that ddPCR may be used for the frequent monitoring of water samples before and after clean-up treatments in order to detect early L. pneumophila growth without waiting the long time necessary for the culture-based methods.

On the basis of our results, the present study represents the starting point for future analyses performed in both water samples and human samples obtained from patients with suspected Legionellosis in order to validate the clinical application of ddPCR for the early and effective detection of L. pneumophila.

Of note, the present study represents an in vitro simulation of L. pneumophila growth and treatment, therefore, it is subjected to some limitations. In particular, here we took into account only L. pneumophila. It is known that in environmental or human samples there are several bacteria which, together with cellular debris and degraded DNA, can interfere with the correct detection of Legionella. However, although this represents a limit for culture methods and for molecular methods based on RT-qPCR, here we demonstrated that ddPCR is not affected by the presence of fragmented DNA or cell debris thanks to the nanopartitions of gene targets and the dilutions of contaminants into thousands of droplets. In this context, other studies support our findings and the use of ddPCR for the detection of bacterial DNAemia during infection or for the monitoring of bacterial load in contaminated samples with PCR inhibitors $(27,28)$.

In conclusion, overall, the results of the present study strongly support the adoption of ddPCR for the effective detection of L. pneumophila in water samples and for the constant monitoring of bacterial load in sites considered at risk, such as the hospital environment. Although this study represents only an in vitro simulation of the Legionella growth, the results obtained encourage the use of ddPCR also in the clinical setting for the evaluation of patients with suspected Legionellosis.

In particular, ddPCR would allow the early detection of any L. pneumophila increment in the contaminated site or in patients thus establishing the efficacy of water and antibiotic treatments, respectively. In this context, the use of ddPCR could have important impacts from both health and socio-economic points of view allowing the reduction of the long times necessary to diagnose L. pneumophila with the standard culture methods. Accordingly, through ddPCR analysis, the structure, whether public or private hospital, will more effectively monitor possible contamination and the efficacy of treatments thus restarting more rapidly their activities with a significant economic benefit.

\section{Acknowledgements}

This study was supported by the Italian League Against Cancer (LILT).

\section{Funding}

No funding was received.

\section{Availability of data and materials}

The data generated and analyzed during the current study are available from the corresponding author on reasonable request.

\section{Authors' contributions}

ML, DAS, MS and LF conceived the study. LF, GG, CMG, GL and CL were involved in the methodologies used and in the validation of methods. LF, GG, CMG, GL and CL performed the formal and statistical analyses. LF and GG wrote the manuscript. ML, DAS and MS revised the manuscript draft. All authors have read and agreed to the published version of the manuscript. 


\section{Ethics approval and consent to participate}

Not applicable.

\section{Patient consent for publication}

Not applicable.

\section{Competing interests}

DAS is the Editor-in-Chief for the journal, but had no personal involvement in the reviewing process, or any influence in terms of adjudicating on the final decision, for this article. The other authors declare that they have no competing interests.

\section{References}

1. Cunha BA, Burillo A and Bouza E: Legionnaires' disease. Lancet 387: 376-385, 2016.

2. Stout JE and Yu VL: Legionellosis. N Engl J Med 337: 682-687, 1997.

3. European Centre for Disease Prevention and Control: Legionnaires' Disease in Europe, Annual Epidemiological Report for 2017. ECDC, Stockholm, 2019.

4. Istituto Superiore di Sanità (ISS): Rapporto Annuale sulla Legionellosi in Italia Nel 2017. Vol 31. ISS, Rome, 2018.

5. Bartram J, Chartier Y, Lee JV, Pond K and Surman-Lee S: Legionella and the prevention of legionellosis. World Health Organization Press, Geneva, 2007.

6. Fliermans CB, Soracco RJ and Pope DH: Measure of Legionella pneumophila activity in situ. Curr Microbiol 6: 89-94, 1981.

7. Abdel-Nour M, Duncan C, Low DE and Guyard C: Biofilms: The stronghold of Legionella pneumophila. Int J Mol Sci 14: 21660-21675, 2013.

8. Liu Z, Lin YE, Stout JE, Hwang CC, Vidic RD and Yu VL: Effect of flow regimes on the presence of Legionella within the biofilm of a model plumbing system. J Appl Microbiol 101: 437-442, 2006

9. van Heijnsbergen E, Schalk JA, Euser SM, Brandsema PS, den Boer JW and de Roda Husman AM: Confirmed and potential sources of Legionella reviewed. Environ Sci Technol 49: 4797-4815, 2015.

10. Linee guida per la prevenzione ed il controllo della legionellosi. Conferenza Stato-Regioni, nella seduta del 7 maggio 2015. http://www.salute.gov.it/portale/documentazione/p6_2_2_1.jsp? lingua $=$ italiano\&id=2362. Accessed May 13, 2015.

11. ISO 11731:2017. Water Quality-Enumeration of Legionella. International Organization for Standardization, Geneva, 2017. https://www.iso.org/standard/61782.html.

12. Kimura S, Tateda K, Ishii Y, Horikawa M, Miyairi S, Gotoh N, Ishiguro $\mathrm{M}$ and Yamaguchi K: Pseudomonas aeruginosa Las quorum sensing autoinducer suppresses growth and biofilm production in Legionella species. Microbiol Read 155: 1934-1939, 2009.

13. Leoni E and Legnani PP: Comparison of selective procedures for isolation and enumeration of Legionella species from hot water systems. J Appl Microbiol 90: 27-33, 2001.

14. Ta AC, Stout JE, Yu VL and Wagener MM: Comparison of culture methods for monitoring Legionella species in hospital potable water systems and recommendations for standardization of such methods. J Clin Microbiol 33: 2118-2123, 1995.
15. Touron-Bodilis A, Pougnard C, Frenkiel-Lebossé $\mathrm{H}$ and Hallier-Soulier S: Usefulness of real-time PCR as a complementary tool to the monitoring of Legionella spp. and Legionella pneumophila by culture in industrial cooling systems. J Appl Microbiol 111: 499-510, 2011.

16. Bonetta S, Bonetta S, Ferretti E, Balocco F and Carraro E: Evaluation of Legionella pneumophila contamination in Italian hotel water systems by quantitative real-time PCR and culture methods. J Appl Microbiol 108: 1576-1583, 2010.

17. Edagawa A, Kimura A, Doi H, Tanaka H, Tomioka K, Sakabe K, Nakajima C and Suzuki Y: Detection of culturable and nonculturable Legionella species from hot water systems of public buildings in Japan. J Appl Microbiol 105: 2104-2114, 2008.

18. Lee $\mathrm{S}$ and Lee J: Outbreak investigations and identification of Legionella in contaminated water. Methods Mol Biol 954: 87-118, 2013.

19. Arvia R, Sollai M,Pierucci F, Urso C, Massi D and Zakrzewska K: Droplet digital PCR (ddPCR) vs quantitative real-time PCR (qPCR) approach for detection and quantification of Merkel cell polyomavirus (MCPyV) DNA in formalin fixed paraffin embedded (FFPE) cutaneous biopsies. J Virol Methods 246: 15-20, 2017.

20. Hayden RT, Gu Z, Ingersoll J, Abdul-Ali D, Shi L, Pounds S and Caliendo AM: Comparison of droplet digital PCR to real-time PCR for quantitative detection of cytomegalovirus. J Clin Microbiol 51: 540-546, 2013.

21. Filetti V, Falzone L, Rapisarda V, Caltabiano R, Eleonora Graziano AC, Ledda C and Loreto C: Modulation of microRNA expression levels after naturally occurring asbestiform fibers exposure as a diagnostic biomarker of mesothelial neoplastic transformation. Ecotoxicol Environ Saf 198: 110640, 2020.

22. Battaglia R, Palini S, Vento ME, La Ferlita A, Lo Faro MJ, Caroppo E, Borzì P, Falzone L, Barbagallo D, Ragusa M, et al: Identification of extracellular vesicles and characterization of miRNA expression profiles in human blastocoel fluid. Sci Rep 9: 84, 2019.

23. Salemi R, Falzone L, Madonna G, Polesel J, Cinà D, Mallardo D, Ascierto PA, Libra M and Candido S: MMP-9 as a candidate marker of response to BRAF inhibitors in melanoma patients with BRAFV600E mutation detected in circulating-free DNA. Front Pharmacol 9: 856, 2018.

24. Falzone L, Musso N, Gattuso G, Bongiorno D, Palermo CI, Scalia G, Libra M and Stefani S: Droplet digital PCR as the best sensitive assay for the SARS-CoV-2 detection. Int J Mol Med 46: 957-964, 2020

25. Krøjgaard LH, Krogfelt KA, Albrechtsen HJ and Uldum SA: Detection of Legionella by quantitative-polymerase chain reaction (qPCR) for monitoring and risk assessment. BMC Microbiol 11: 254, 2011

26. Shen SM, Chou MY, Hsu BM, Ji WT, Hsu TK, Tsai HF, Huang YL, Chiu YC, Kao ES, Kao PM, et al: Assessment of Legionella pneumophila in recreational spring water with quantitative PCR (Taqman) assay. Pathog Glob Health 109: 236-241, 2015.

27. Ziegler I, Lindström S, Källgren M, Strålin K and Mölling P: 16S rDNA droplet digital PCR for monitoring bacterial DNAemia in bloodstream infections. PLoS One 14: e0224656, 2019.

28. Singh G, Sithebe A, Enitan AM, Kumari S, Bux F and Stenström TA: Comparison of droplet digital PCR and quantitative PCR for the detection of Salmonella and its application for river sediments. J Water Health 15: 505-508, 2017.

(i) $(-)$ This work is licensed under a Creative Commons Attribution-NonCommercial-NoDerivatives 4.0 International (CC BY-NC-ND 4.0) License. 